East African Medical Journal Vol. 81 No. 3 March 2004

COMPARISON OF CALCULATED AND DIRECT LOW DENSITY LIPOPROTEIN CHOLESTEROL DETERMINATIONS IN A ROUTINE LABORATORY A. A. Amayo, MBChB., MMed., Lecturer, Department of Clinical Chemistry, College of Health Sciences, University of Nairobi, P.O Box 19676, Nairobi, Kenya, and S. Kirera HND (Biochemistry) Technologist, Mater Hospital Laboratory, P.O Box 30325, Nairobi, Kenya

Request for reprints to: Dr. A. A Amayo, Department of Clinical Chemistry, College of Health Sciences, University of Nairobi, P.O Box 19676, Nairobi, Kenya

\title{
COMPARISON OF CALCULATED AND DIRECT LOW DENSITY LIPOPROTEIN CHOLESTEROL DETERMINATIONS IN A ROUTINE LABORATORY
}

\author{
A. A. AMAYO and S. KIRERA
}

\begin{abstract}
Background: Low density lipoprotein cholesterol (LDL-C) concentrations form the basis for treatment guidelines established for hyperlipidaemic patients. LDL-C concentrations are commonly calculated using the Friedwald formula (FF) which has several limitations. Recently, direct methods for LDL-C estimation have been developed which are suitable for routine laboratories.

Objective: To compare serum LDL-C concentrations determined by a direct assay and the Friedwald formula.

Design: Cross-sectional study.

Setting: Mater Hospital Laboratory, Nairobi, Kenya. Methods: The clinical performance of the two methods was evaluated by analysing 211 fresh plasma samples from fasting adult patients. The samples were divided into four groups-normolipidaemic; and Types IIa, IIb and IV hyperlipidaemias.

Results: The Friedwald formula (FF) correlated best with the direct assay in the normolipidaemic samples $(r=0.879 ; y=0.468+0.852 x)$. Direct LDL-C values were significantly lower than the FF in the Type IIa hyperlipidaemia samples (paired differences $0.38 \pm 0.62$ ). There was only $65 \%$ agreement between the two methods in the borderline high LDL-C group of the National Cholesterol Education Program (NCEP) classification (LDL-C 3.36 - $4.14 \mathrm{mmol} / \mathrm{L}$ ).

Conclusion: There is lack of agreement between the FF and the Abbott direct LDL$\mathrm{C}$ assay. If the two methods are used interchangeably, there may be confusion in the classification and control of lipid lowering medication for patients with hyperlipidaemia.
\end{abstract}

\section{INTRODUCTION}

The association between increased concentrations of low-density lipoprotein cholesterol (LDL-C) and increased risk of coronary artery disease (CAD) has been clearly demonstrated in clinical and epidemiological studies(1-3), and intervention with dietary or drug therapy to lower LDL-C has been shown to decrease morbidity and cardiovascular mortality $(4,6)$.

Because of the causal role LDL-C plays in the development of atherosclerosis, blood LDL-C concentration is an important criterion for diagnosis and treatment of patients with hyperlipidaemia (HPL). The accepted "gold" standard method for blood LDL-C estimation is the beta - quantification (BQ-LDL) which is an expensive, labour intensive method and not generally available in routine laboratories $(7,8)$.

Most clinical laboratories have therefore depended on calculations of LDL-C using the Friedwald formula (FF) which is based on three independent measurements: total cholesterol (TC), HDL-cholesterol (HDL-C) and triglycerides (TG)(9). This equation assumes that the amount of cholesterol in very-low density lipoprotein
(VLDL) can be estimated by dividing the serum TG by a factor of 5. The use of the FF has shortcomings: firstly, combining three measurements increases analytical variability; secondly, the formula has been shown to be invalid in samples with high triglyceride concentrations and can therefore only be used in fasting blood samples. Thirdly the assumption that the relationship between cholesterol and triglyceride in VLDL is constant has been shown to be inaccurate in some hyperlipidaemias $(10,11)$.

Several direct assays for LDL-C estimation have recently been developed and the kits are available for use by routine laboratories. There are reports of differences between LDL-C values calculated using the FF and those obtained by direct assays(12-15). Since some laboratories continue to use the FF and others use the two methods interchangeably $(16,17)$, significant variations in the LDL$\mathrm{C}$ results obtained by the different methods may create confusion in the risk stratification of patients and treatment follow-up as the use of LDL-C measurements in routine laboratories increases. In this study LDL-C was calculated using the FF and the values were compared with those obtained from a direct LDL-C assay used routinely in this laboratory. 


\section{MATERIALS AND METHODS}

Samples: Plasma samples from 211 adults received at the Mater Hospital Laboratory, Nairobi were used. Only those where a full fasting lipid profile was requested were considered. Sampies with fasting TG concentrations $>4.5 \mathrm{mmol} / \mathrm{L}$ were excluded from the FF calculations because the equation has been clearly shown to be invalid in hypertriglyceridaemic samples $(10,11)$.

Quantification of lipids: All the lipid analyses were performed on the Alcyon 300i (Abbott Park IL). Total cholesterol and TG were measured enzymatically with the appropriate reagents from Abbott Diagnosis ( Coefficients of variation (CV) $2.4 \%$ and $5.6 \%$ for $\mathrm{TC}$ and $\mathrm{TG}$ respectively). HDL-C: A homogeneous direct HDL-C method was used (Abbott reagent list 8D42-02). This assay utilizes a unique detergent to solubilise only the high density lipoptrotein particles, thus releasing HDL-C to react with cholesterol esterase and cholesterol oxidase in the presence of chromogens to produce colour. Analysis was done on the Alcyon 300i according to the manufacturer's protocol (CV 5.2\%).

$L D L-C$ : A homogeneous direct LDL-C method was used (Abbott reagent list 8D45-02). The assay uses two reagents. Reagent 1 solubilises only the non LDL particles. The cholesterol released is consumed by cholesterol esterase and cholesterol oxidase in a non colour forming reaction. Reagent 2 solubilises the remaining LDL particles and a chromogenic coupler allows for colour development. Analysis was done on the Alcyon 300i according to the manufacturer's protocol (CV $4.1 \%$ ). All lipid analyses were performed within three hours of sample collection.

Friedwald calculation: LDL-C was estimated as follows LDL-C $=\mathrm{TC}-\left({ }^{\mathrm{TG}} / 2.17\right)-(\mathrm{HDL}-\mathrm{C})(8)$.

Comparison between methods: For this purpose, samples were classified into four groups according to their TC and TG concentrations (i) Normolipidaemia defined as TC $\leq 6.2$ $\mathrm{mmol} / \mathrm{L}$ and $\mathrm{TG} \leq 2.66 \mathrm{mmol} / \mathrm{L}$; (ii) Type II a hyperlipidaemia (HPL) as TC $>6.2 \mathrm{mmo} / \mathrm{L}$ and $\mathrm{TG}<2.66 \mathrm{mmo} / \mathrm{L}$; (iii) Type IIb HPL as TC $>6.2 \mathrm{mmol} / \mathrm{L}$ and TG $>2.66 \mathrm{mmol} / \mathrm{L}$ and (iv) Type IV HPL as TC $\leq 6.2 \mathrm{mmol} / \mathrm{L}$ and TG $>2.66 \mathrm{mmol} / \mathrm{L}$. Statistical analysis: All data were entered in a personal computer. Descriptive statistics (means, standard deviations (SD) and CVs) were calculated with Microsoft Excel (Microsoft). Mean values for LDL-C by the two methods were compared by paired student's t-tests. Linear relationships were determined from standard Pearson correlation coefficients by linear regression analyses using SPSS (VER 10.0). To assess the degree of agreement between the methods, the graphical procedure outlined by Bland and $\operatorname{Altman}(18)$ was used. A scattergram of the methods versus the difference between the two methods was prepared. Differences were considered significant at $\mathrm{p}<0.05$.

\section{RESULTS}

The lipid and lipoprotein concentrations, and correlation results are summarised in Table 1 . The overall mean for direct LDL-C was $3.24 \mathrm{mmo} / \mathrm{L}$ which compared well with for the FF $(3.30 \mathrm{mmo} / \mathrm{L})$. The correlation coefficient for this association was $r=0.891$. However, when the four groups were analysed separately, differences were noted between the two methods in some groups. As in shown in linear regression analysis (Figure 1 Top panels), the best correlation between the two methods was seen with the normolipidaemic samples: $r=$ 0.879 , slope $=0.82$ (Figure 1A). Good correlation was also found among the Type IIb HPL samples: $r=0.886$, slope $=0.758$ but this was not statistically significant (Figure 1C). Among Type IIa HP-L samples, results obtained by the two methods showed significant deviation from the line of identity: $r=0.525$, slope $=0.537$ ( Figure 1B). Deviation was also seen in the Type IV HPL sample but it did not attain statistical significance (Figure 1D).

Table 1

Summary of Cholesterol, TG'S, HDL-C and LDL-C; and correlation between calculated and direct LDC-C among the groups $(a)$

\begin{tabular}{lllll}
\hline & $\begin{array}{l}\text { Normolipidaemia } \\
152\end{array}$ & $\begin{array}{l}\text { Type IIa HPL } \\
32\end{array}$ & $\begin{array}{l}\text { Type IIb HPL } \\
15\end{array}$ & $\begin{array}{l}\text { Type IV HPL } \\
12\end{array}$ \\
\hline Total cholestero(mmol/L) & $4.9 \pm 0.825$ & $6.9 \pm 0.675$ & $5.2 \pm 0.746$ & $6.8 \pm 0.611$ \\
TG mmol/L & $1.28 \pm 0.541$ & $1.63 \pm 0.574$ & $3.23 \pm 0.483$ & $3.46 \pm 1.00$ \\
HDL mmol/L & $1.28 \pm 0.343$ & $1.40 \pm 0.260$ & $1.04 \pm 0.273$ & $1.22 \pm 0.277$ \\
Direct LDL-C mmol/L & $2.97 \pm 0.766$ & $4.40 \pm 0.628$ & $2.86 \pm 0.670$ & $4.28 \pm 0.631$ \\
Calc. LDL-C mmol/L & $3.0 \pm 0.743 \mathrm{~b}$ & $4.78 \pm 0.643 \mathrm{~b}$ & $2.66 \pm 0.573$ & $4.03 \pm 0.602$ \\
Paired diff. mmol/L & $0.03 \pm 0.371$ & $0.38 \pm 0.62$ & $0.20 \pm 0.310$ & $0.34 \pm 0.526$ \\
Correlations calculated LDC-C.direct LDC-C & & & 1.771 \\
Intercept & 0.468 & 2.419 & 0.495 & 0.638 \\
Slope0.852 & 0.537 & 0.758 & 0.527 & 0.12 \\
$\mathrm{r}$ & 0.879 & 0.525 & 0.886 & \\
$\mathrm{p}$ & 0.00 & 0 & 0.017 & \\
\hline
\end{tabular}

a Results are given as mean $\pm \mathrm{SD}$

$\mathrm{P}<0.05$ in relation to direct LDL-C

Table 2

Agreement in classification of $L D L-C$ by the $F F$ compared with the direct assay at the NCEP cutpoints of 3.37 and $4.14 \mathrm{mmol} / \mathrm{L}$

\begin{tabular}{lll}
\hline & Agreement $(\%)$ \\
\hline$<3.37 \quad(\mathrm{n}=119)$ & 91 \\
$3 . .37-4.13(\mathrm{n}=61)$ & 65 \\
$\geq 4.14 \quad(\mathrm{n}=31)$ & 94 \\
\hline
\end{tabular}


Figure 1

Correlations (top panels) and Bland-Altman plots

(bottom panels)

1a: $L D L-C$ in normolipidaemic

1b: Type IIa HP

1d: Type IV HPL samples 
Agreement between the two methods was assessed using the Bland - Altman graphical technique(18). The degree of agreement is indicated by the bias, estimated by the mean and SD of the differences. The direct LDL$\mathrm{C}$ values were significantly lower than those obtained by the FF in the type IIa HPL samples (paired differences $-0.38 \pm 0.62$ ). Thus in the Bland - Altman plot, most of the points lie below zero on the $\mathrm{x}$ - axis (Figure 1B, Bottom panel). There were also differences among the type IIb and IV HPL samples as most points on the Bland Altman plots lie on one side of the mean, but these were not statistically significant (Figure 1C, ID). The good correlation in the normolipidaemic samples was verified by the Bland Altman plots which showed an equal distribution of the points around the mean (paired differences $-0.03 \pm 0.371$ ) (Figure 1A, Bottom panel).

Classification of samples with the NCEP cut off points: The National Cholesterol Education Program (NCEP) Adult Treatment Program II (ATP II) of 1994 laid down LDL-C values of <3.36, 3.36 - 4.14, and $\geq 4.14$ as cutoff points for classifying patients into normal, borderline and high LDL-C (8). We studied the agreement in classification by the two methods into the three categories with CLDL-C values obtained by the FF coinciding within $10 \%$ of the direct LDL-C values. As seen in Table 2, there is considerable lack of agreement among the samples in the borderline high category $(35 \%)$. The level of agreement in the other two categories is high $(>90 \%)$.

\section{DISCUSSION}

LDL-C is a key factor in the pathogenesis of premature coronary disease(1-3). Blood LDL-C concentration is used to assess cardiovascular risk and effectiveness of cholesterol lowering regimens $(4,6)$. The accuracy and precision of the method used to estimate blood LDL-C is therefore very important. There is evidence that there is an increase in risk factors for CAD among Africans(19-21), which will increase the demand for blood lipid analyses in routine clinical laboratories. Until recently, most routine laboratories used the FF to calculate LDL-C concentrations. Several direct assays for LDL-C estimation have now been developed which are adaptable to automatic analyzers in routine laboratories.

The findings of this study show that there is good correlation between the Abbott direct homogeneous assay and the FF in the normolipidaemic samples. The two methods were however not in complete agreement as is shown by examination of the Bland Altman plots. Among the hyperlipidaemic samples, there were significant differences in the type ILA HPL samples, the paired differences indicating a positive bias in the Friedwald calculation as compared to the direct LDL$\mathrm{C}$ assay. There were also differences among the type IIb and type IV HPL samples but these did not attain statistical significance possibly because of the comparatively small sample sizes in these two groups.
Previous studies have also reported differences between calculated LDL-C and other methods for LDL$\mathrm{C}$ estimation in various hyperlipidaemias(12-14). Matas and colleagues found significant variation between calculated and measured LDL-C in a group of patients with liver cirrhosis (mean SD: $3.01+1.03$ and $2.59+$ 0.99; $\mathrm{p}<0.001$ for direct and calculated LDL-C respectively), despite the fact that none of the patients had marked hypertriglyceridaemia(12). They attributed the discrepancy to an abnormal VLDL particle in which the cholesterol component was significantly decreased. The FF assumes that most of the circulating triglyceride is carried in VLDL, and that the relationship between the cholesterol and TG in this fraction is constant. This assumption has been demonstrated to be inaccurate in several hyperlipidaemias(22). The particles generally called VLDL are a heterogeneous mix of VLDL, chylomicron remnants and VLDL remnants. The percentage of cholesterol across this range of particles varies significantly depending on the TG concentration. Errors in calculating the cholesterol in the particles will ultimately reflect as an error in the calculated LDL$\mathrm{C}(14)$. It has therefore been recommended that the FF should be used with caution in several conditions associated with hyperlipidaemia, including diabetes mellitus, nephrotic syndrome and hepatopathy, even if triglycerides are less than $4.5 \mathrm{mmol} / \mathrm{L}(14,23)$. The samples used in this study were from a heterogeneous adult population with various underlying disorders some of which may cause such alterations in the concentration and composition of the VLDL particles leading to the bias observed in the type IIa HPL samples. It is possible that with a larger sample size, lack of agreement between the two methods would also be found in the samples with types IIb and IV HPL.

While it is unlikely that different methods will agree exactly, the question is whether the magnitude of the differences will impact clinical judgment. The NCEP ATP II (1994) LDL-C cut-off concentrations of 3.36 $\mathrm{mmo} / \mathrm{L}$ and $4.13 \mathrm{mmol} / \mathrm{L}$ are important parameters in therapeutic decision making. Reporting a patient's LDL$\mathrm{C}$ above or below the conventional cut-off conveys a message about risk stratification and an incorrect classification could lead to inappropriate treatment. This study showed $>90 \%$ agreement in classification for the $<3.36 \mathrm{mmol} / \mathrm{L}$ and $>4.13 \mathrm{mmo} / \mathrm{L}$ groups but only $65 \%$ agreement in the middle $3.36-4.14 \mathrm{mmo} / \mathrm{L}$ group.

Previous LDL-C method comparison studies have also reported higher rates of disagreement in this borderline high risk group than in the other two groups(13-15). Esteban-Salan and colleagues reported that although the FF correctly classified $95 \%$ of patients with normolipidaemia, only $76 \%$ of the rest were correctly classified. Direct methods classified $>90 \%$ of patients in both groups(14). These discrepancies in classification would be of concern to the physician who must interpret LDL-C measurements from different laboratories using different methods. 
These findings invite the question as to which of the two methods is more reliable. The only way to answer this would be to compare the two methods against the reference BQ method. However, because the FF has several sources of error from several measurements but the direct assay has only one source of error from one measurement, it might be reasonable to question the reliability of the FF. Higher accuracy of direct LDL$\mathrm{C}$ assays over $\mathrm{FF}$ in secondary hyperlipidaemias has also been reported(12-14,24).

In conclusion, this study shows lack of agreement between LDL-C estimated by a direct assay and the FF in HPL samples in a routine diagnostic laboratory. There were previous recommendations that direct LDL-C estimation and the FF should be used interchangeably to minimise laboratory costs $(16,17)$, the FF for the initial classification as a full lipid profile is usually required and the direct LDL-C for treatment follow up. There should be caution about adopting this recommendation in a routine laboratory for two reasons. First the routine laboratory handles samples from patients with a wide range of clinical conditions some of which may affect the VLDL composition with attendant errors in the FF. Secondly the adequacy of the FF is governed by strict adherence to the NCEP criteria for TC, TG and HDLC measurements(8).

Small routine laboratories may not always meet the NCEP performance standards and this may decrease the reliability of the FF. Interchanging of the methods may therefore be associated with discordant serial LDL-C results, which may cause confusion in risk assessment as well as follow-up of patients. This may ultimately lead to several repeat analyses and additional costs. Routine diagnostic laboratories should instead maintain one method of LDL-C estimation to ensure internal standardization. This will enable them to keep a constant vigilance on the quality of the results reported so that they are transferable across laboratories.

\section{ACKNOWLEDGEMENTS}

To the administration of the Mater Hospital for allowing this work to be published, and Ms. Evangeline Karimi for secretarial services.

\section{REFERENCES}

1. Gordon, T., Kannel, W.B., Castelli, W.P., and Dawber, T.R. Lipoproteins cardiovascular disease and death. The Framingham study. Arch. Intern. Med. 1981; 141:1128-1131.

2. Grundy, S.M. Role of low-density lipoproteins in atherogenesis and development of coronary heart disease. Clin. Chem. 1995; 41:139-146.

3. Kannel, W.B., Nekton, J.D., Wentworth, D., et al., for the MRFIT Research Group. Overall and coronary heart disease mortality rates in relation to major risk factors in $325 ; 348$ men screened for the Multiple Risk Factor Intervention Trial MRFIT. Amer. Heart J. 1986; 112:825-836.

4. Lipid Research Clinics Program. The Lipid Research Clinics coronary primary prevention trial results: II. The relationship of reduction in incidence of coronary heart disease to cholesterol lowering. J. Amer. Med. Assoc. 1984; 251:365-374.

5. Scandinavian Simvastatin Survival Study Group. Randomized trial of cholesterol lowering in 4,444 patient with coronary heart disease. The Scandinavian Simvastatin Survival Study (4S). Lancet. 1994; 344:1383-1389.

6. Sacks, F.M., Pfeffer, M.A., Moye, L.A., Rouleau, J.L. et al. The effect of pravastatin on coronary events after myocardial infarction in patients with average cholesterol levels. New Engl. J. Med. 1996; 335:14: 1001-1009.

7. DeLong, D.M., Delong, E.R., Wood, P.D., Lippel, K., and Rifkind, B.M. A comparison of methods for the estimation of plasma low- and very low-density lipoprotein cholesterol: the lipid research clinics prevalence study. J. Amer. Med. Assoc. 1986; 256:2372-2377.

8. Bachorik, P.S. and Ross, J.W. National cholesterol education program recommendations for measurement of low density lipoprotein cholesterol: executive summary. Clin. Chem. 1995; 41:1414-1420.

9. Friedewald, W.T., Levy, R.I., and Fredrickson, D.S. Estimation of concentration of low-density lipoprotein cholesterol in plasma without use ofthe ultracentrifuge. Clin. Chem. 1972; 38: $150-160$.

10. Rifai, N., Warnick, G.R., McNamara, J.R., Belcher, J.D., Grinstead, J.F., and Frantz, I.D. Measurement of low-densitylipoprotein cholesterol in serum: a status report (Review). Clin. Chem. 1992; 38:150-160.

11. McNamara, J.R., Cohn, J.S., Wilson, P.W.F., and Schaefer, E.J. Calculated values for low-density lipoprotein in the assessment of lipid abnormalities and coronary disease risk. Clin. Chem. 1990; 36:36-42.

12. Matas, C., Cabre, M., La Vilie, A., et al. Limitations of the Friedewald formula for estimating low-density lipoprotein cholesterol in alcoholics with liver disease. Clin. Chem. 1994: 40:404-406.

13. Maitra, A., Hirany, S.V. and Jialal, I. Comparison of two assays for measuring LDL cholesterol. Clin. Chem. 1997; 43:6: 1040-1047.

14. Esteban-Salan, M., Guimon-Bardesi, A., Viuda-Unzueta, J.M., Azcarate-Ania, M.N., et al. Analytical and clinical evaluation of two homogeneous assays for LDL-cholesterol in Hyperlipidemic patients. Clin. Chem. 2000; 46:1121-1131.

15. Harris, N. Neufeld, E.J., Newburger, J.W., Ticho, B. and Baker, A. Analytical performance and clinical utility of a direct LDL-cholesterol assay in a hyperlipidaemic pediatric population. Clin. Chem. 1996; 42:1182-1188.

16. Schectman, G., Patsches, M., and Sasse, E.A. Variability in cholesterol measurements: comparison of calculated and direct LDL cholesterol determinations. Clin. Chem. 1996: 42: 732-737.

17. Krause, B.R., Schork, N.J., Kieft, K.A., Smith, M.P. et al. High correlation but lack of agreement between direct highperformance gel chromatography analysis and conventional indirect methods for determining lipoprotein cholesterol. Clin. Chem. 1996; 42:1996-2001.

18. Bland, J.M., and Altman, D.G. Statistical methods for assessing agreement between two methods of clinical measurement. Lancet. 1986; 1:307-310.

19. Yonga, G.O., Ogola E.N. and Juma F.D. Cardiovascular risk factor profiles in mild to moderate hypertensives at Kenyatta Hospital. East Afr. Med. J. 1993; 70:693.

20. Amoah, A.G.B. Spectrum of Cardiovascular disorders in a National referral centre, Ghana. East Afr. Med. J. 2000; 77:648-653.

21. Gebre-Yohannes, A. and Rahlenbeck, S.I. Coronary heart disease risk factors among blood donors in Northwest Ethiopia. East Afr. Med. J. 1998; 75:495-500.

22. Jialal, I.A. practical approach to the laboratory diagnosis of dyslipidemia. Amer J. Clin. Pathol. 1996; 106:128-138.

23. Rubies-Prat, J., Revere, R.J., Senti, M., Pedro-Botet, J. et al. Calculated low-density lipoprotein cholesterol should not be used for management of lipoprotein abnormalities in patients with diabetes mellitus. Diabetes Care. 1993; 16:10811086.

24. Hirany, S.V., Li, D. and Jialal, I. A more valid measurement of LDL cholesterol in diabetic patients. Amer. J. Med. 1997; 102:48-53. 